\title{
SACARIFICAÇÃO DE DIFERENTES RESÍDUOS AGROINDUSTRIAIS PELO EXTRATO ENZIMÁTICO BRUTO PRODUZIDO POR Trichoderma reesei NRRL 3652 EM CASCA DE SOJA
}

V. ASTOLFI ${ }^{1}$, A. L. ASTOLFi ${ }^{1}$, C. E. V. BUSTAMANTE ${ }^{1}$, L. H. CULAU ${ }^{1}$, J. A. do NASCIMENTO ${ }^{1}$, E. RIGO ${ }^{2}$, M. DI LUCCIO ${ }^{3}$, H. TREICHEL ${ }^{4}$

${ }^{1}$ Universidade Regional Integrada - Campus de Erechim

${ }^{2}$ Universidade do Estado de Santa Catarina, Departamento de Engenharia de Alimentos Campus de Pinhalzinho

${ }^{3}$ Universidade Federal de Santa Catarina, Departamento de Engenharia Química e

Engenharia de Alimentos

${ }^{4}$ Universidade Federal Fronteira Sul - Campus de Erechim

E-mail para contato: vivi_ast@yahoo.com.br

\begin{abstract}
RESUMO - Atualmente tem se buscado esforços para o desenvolvimento e a implementação de tecnologias para produção de etanol celulósico a partir de resíduos agrícolas com baixo valor agregado, como bagaço de cana, casca de soja, resíduos de madeira, palha de arroz, palha de trigo, entre outros. A biomassa vegetal é constituída principalmente de celulose, hemicelulose e lignina, sendo a celulose o polímero mais abundante. A hidrólise enzimática é um importante passo para a bioconversão da fração celulósica e hemicelulósica de resíduos lignocelulósicos em açúcares fermentescíveis. Neste estudo, o objetivo foi investigar a sacarificação de casca de soja, bagaço de cana e casca de arroz, utilizando-se o extrato enzimático bruto obtido da fermentação em estado sólido de $T$. reesei NRRL 3652 em casca de soja, com finalidade da obtenção de açúcares fermentescíveis. Obteve-se maior rendimento de glicose com casca de arroz, no entanto é importante destacar que para que a sacarificação da biomassa lignocelulósica apresente elevados rendimentos, faz-se necessário a realização de processos de pré-tratamento, isto porque a lignina e hemicelulose presente na parede da célula vegetal formam uma barreira para ação enzimática.
\end{abstract}

\section{INTRODUÇÃO}

As celulases e hemicelulases são enzimas que constituem um complexo capaz de atuar sobre materiais lignocelulósicos, promovendo sua hidrólise. Estas enzimas são biocatalisadores altamente específicos que atuam em sinergia para a liberação de açúcares fermentescíveis, despertando interesse industrial para produção em larga escala de bioetanol de segunda geração (JURUTU e WU, 2014).

Para bioconversão de biomassa lignocelulósica em açúcares, eficazes e econômicos pré-tratamentos devem ser desenvolvidos de tal forma que um alto rendimento de açúcares fermentáveis possa ser obtido na subsequente etapa de hidrólise enzimática; condições de pré- 
tratamento também devem ser balanceados para evitar a formação de inibidores da hidrólise e processos de fermentação da biomassa (SANCHEZ e CARDONA, 2008). Além disso, o baixo custo, a estabilidade e a eficiência das enzimas também são de suma importância, isto porque o alto custo de enzimas restringe seu uso em aplicações de larga escala para a conversão de materiais lignocelulósicos.

Assim, o objetivo deste trabalho foi avaliar a potencialidade de sacarificação de casca de soja, bagaço de cana e casca de arroz, utilizando-se o extrato enzimático bruto obtido da fermentação em estado sólido de T. reesei NRRL 3652 em casca de soja.

\section{MATERIAL E MÉTODOS}

O extrato enzimático bruto obtido da condição experimental ótima da fermentação em estado sólido de $T$. reesei NRRL 3652 em casca de soja foi filtrado em bomba de vácuo com o auxilio de papel filtro e continha atividade de $6,35 \mathrm{U} . \mathrm{g}^{-1} ; 4,30 \mathrm{U} . \mathrm{g}^{-1} ; 1228,52 \mathrm{U}_{\mathrm{g}} \mathrm{g}^{-1}$ para FPase, CMCase e xilanase, respectivamente. A sacarificação enzimática da casca de soja, bagaço de cana e casca de arroz (autoclavados por 15 minutos a $121^{\circ} \mathrm{C}$ ) sem nenhum tratamento foi realizada conforme Liu et al. (2011) com algumas modificações. Os ensaios foram realizados em frascos erlenmeyers no qual, $2 \mathrm{~g}$ do respectivo resíduo lignocelulósico foi adicionado em $100 \mathrm{~mL}$ da mistura reacional, contendo: $94 \mathrm{~mL}$ de tampão citrato de sódio $0,05 \mathrm{M} \mathrm{pH} 5,3 ; 1 \mathrm{~mL}$ de tetraciclina $\left(40 \mu . \mathrm{mL}^{-1}\right)$, com o intuito de inibir a contaminação microbiana e $5 \mathrm{~mL}$ do extrato enzimático bruto. As reações foram realizadas em agitador orbital a $150 \mathrm{rpm}$ e $50{ }^{\circ} \mathrm{C}$ por um período de 7 dias, do qual, amostras foram retiradas da mistura reacional em intervalos de 24 horas e a liberação de açúcares redutores (AR) e glicose na hidrólise foram estimados usando o método do DNS e GOD-POD, conforme item 2.1 e 2.2 , respectivamente.

Ensaios sem substrato lignocelulósico e extrato enzimático bruto (enzima) foram realizados como controle. A \% de sacarificação foi quantificada conforme a Equação 1:

$$
\text { Sacarificação }(\%)=\frac{A_{L} \times 0,9}{P} \times 100
$$

Sendo que:

$A_{L}=$ açúcar liberado $(\mathrm{g})$

$0,9=$ fator de correção para compensar adição de uma molécula de água durante a hidrólise $P=$ polissacarídeos no substrato lignocelulósico $(\mathrm{g})$.

\subsection{Quantificação dos açúcares redutores (AR)}

Para a quantificação dos $\mathrm{AR}$, adicionou-se $0,5 \mathrm{~mL}$ das amostras a serem quantificadas em 0,5 mL de DNS (ácido 3,5-dinitrosalicílico) (MILLER, 1959). Os tubos foram mantidos em um banho a $95^{\circ} \mathrm{C}$ por 5 minutos para desenvolvimento da coloração avermelhada. Após este tempo reacional, as amostras foram resfriadas imediatamente pela imersão dos tubos em um banho de gelo fundente, adicionando-se em seguida $8 \mathrm{~mL}$ da solução estabilizante de tartarato de sódio e potássio tetra-hidratado. Os tubos foram agitados para homogeneizar a solução e as leituras da absorbância, efetuadas no espectrofotômetro a 540 ๆm. Para zerar o espectrofotômetro, preparou-se um tubo onde o volume da amostra foi substituído por água 
destilada. Para determinar a concentração dos AR em cada uma das amostras analisadas utilizou-se a curva-padrão de glicose.

\subsection{Determinação da concentração de glicose}

A concentração de glicose foi determinada de acordo com o método enzimático Glicose Oxidase/Peroxidase (GOD-POD), proposto por Keston (1956). Trata-se de um kit enzimático contendo um reativo monoreagente pronto para uso, além de uma solução padrão de glicose com a concentração de $100 \mathrm{mg}$. $\mathrm{dL}^{-1}$. O método baseia-se na oxidação enzimática da glicose através da enzima glicose oxidase (GOD) resultando em peróxido de hidrogênio, o qual é subsequentemente usado na geração da coloração rosada pela peroxidase (POD).

Para a quantificação da glicose, adicionou-se $30 \mu \mathrm{L}$ de cada uma das amostras em estudo em 1,0 mL do monoreagente. Os tubos foram mantidos em um banho termostático a $37{ }^{\circ} \mathrm{C}$ por 10 minutos para que houvesse o desenvolvimento da coloração rosada e em seguida, efetuou-se a leitura da absorbância em espectrofotômetro a 505 ๆm.

Os tubos do branco da reação e do padrão de glicose foram feitos juntamente com os tubos das amostras. Para o branco adicionou-se $30 \mu \mathrm{L}$ de água destilada em 1,0 mL do reagente enzimático e para o tubo padrão adicionou-se $30 \mu \mathrm{L}$ da solução padrão de glicose (100 mg. dL $\left.{ }^{-1}\right)$ em 1,0 mL do monoreagente enzimático. Outra observação relevante do fabricante é que, quando a leitura do branco apresenta absorbância acima de 0,300, o monoreagente deve ser descartado, já que isso indica uma deterioração do reativo de trabalho. A cor final da reação deverá ser estável por 60 minutos. Para determinar a concentração de glicose utilizou-se a solução padrão como calibrador e os cálculos foram realizados através da Equação 2.

$$
\text { Glicose }\left(m g . d L^{-1}\right)=\frac{\text { Absorbância do teste }}{\text { Absorbância do padrão }} \times 100
$$

\section{RESULTADOS E DISCUSSÃO}

A sacarificação enzimática é dependente tanto, das características da biomassa quanto da eficácia das enzimas. Para tanto, a eficácia do extrato enzimático bruto produzido por T. reesei NRRL 3652 foi testada na sacarificação de casca de arroz, casca de soja e bagaço de cana sem tratamentos, com a finalidade da obtenção de açúcares redutores (AR) e glicose.

Sukumaran et al. (2009) demonstraram que a concentração de enzima pode ter um efeito menor sobre a hidrólise da celulose em comparação com o efeito do tempo de reação. Assim de acordo com Liu et al. (2011) um período de sete dias foi definido no ensaio de sacarificação de biomassa, a fim de se obter açúcares fermentescíveis. As Figuras 1 e 2 apresentam os rendimentos de AR e glicose, respectivamente, liberados experimentalmente por um período de $170 \mathrm{~h}$ de reação. Como pode ser visto na Figura 1, a liberação de AR aumentou rapidamente na fase inicial ( 0 e $24 \mathrm{~h}$ ), principalmente, para casca de arroz e casca de soja, e a porcentagem mais elevada $(16,8 \%)$ foi obtida depois de 120 horas de reação utilizando-se casca de soja. Assim sendo, a quantidade total de AR liberados a partir de $1 \mathrm{~g}$ de 
casca de soja catalisado pelas enzimas presentes no extrato enzimático bruto foi de $0,16 \mathrm{~g}$. $\mathrm{g}^{-1}$ de substrato seco.

Figura1 - Perfil da produção de açúcares redutores na sacarificação de resíduos agroindustriais usando extrato bruto enzimático produzido por T. reesei NRRL 3652 em casca de soja.

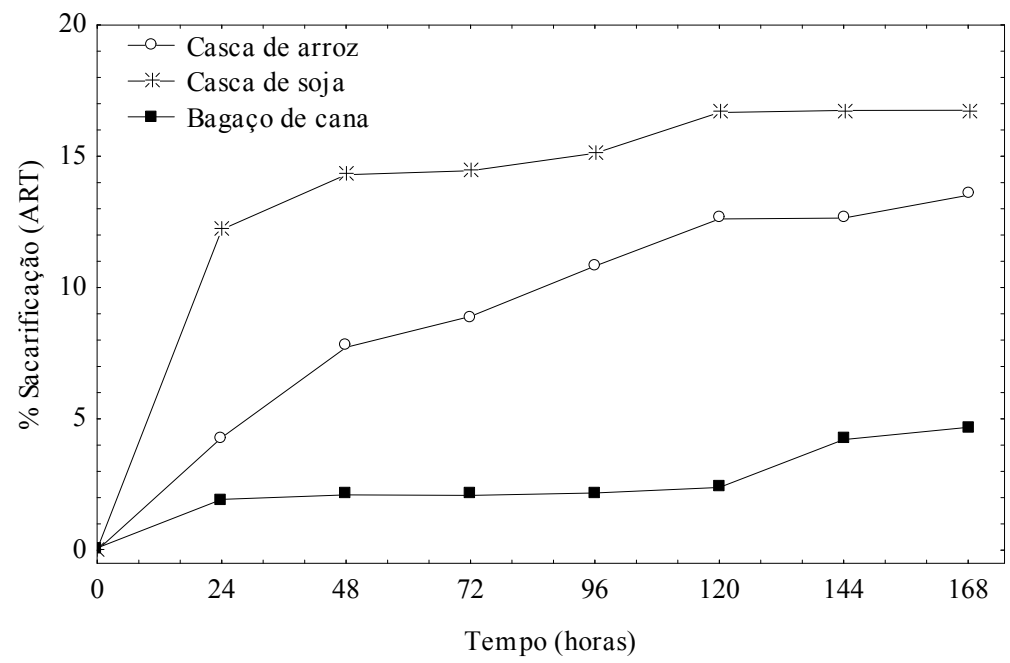

Raghuwanshi et al. (2014) utilizaram o extrato enzimático bruto produzido a partir da cepa selvagem Trichoderma asperellum RCK2011 e a cepa mutante, Trichoderma asperellum SR1-7 para sacarificação de sabugo de milho, bagaço de cana e palha de trigo pré-tratados e não tratados. Os rendimentos de AR foram de $0,53 \mathrm{~g}$. $\mathrm{g}^{-1}, 0,33 \mathrm{~g} \cdot \mathrm{g}^{-1}$ e $0,29 \mathrm{~g}$. $\mathrm{g}^{-1} \mathrm{de}$ substrato seco, respectivamente, do qual, isto foi 1,6 vezes mais elevado do que a estirpe do tipo selvagem.

Deswal et al. (2011) utilizaram o extrato enzimático bruto de Fomitopsis sp. RCK2010 para sacarificação em palha de trigo e palha de arroz pré-tratada alcalinamente. Os rendimentos de AR para palha de trigo e palha de arroz foram de $0,214 \mathrm{~g}$. $\mathrm{g}^{-1}$ e $0,157 \mathrm{~g}^{\mathrm{g}} \mathrm{g}^{-1} \mathrm{de}$ substrato seco, respectivamente, porém rendimentos abaixo de $0,10 \mathrm{~g}$. $\mathrm{g}^{-1}$ de substrato seco foram obtidos quando os substratos não foram tratados.

Liu et al. (2011) obtiveram rendimentos de AR de 0,45 g. $\mathrm{g}^{-1}$ de substrato seco a partir da hidrólise de palha de milho utilizando o extrato enzimático bruto produzido por A. fumigatus Z5.

Como pode ser observado na Figura 2, a casca de arroz apresentou o melhor rendimento em glicose liberada principalmente, nas primeiras 72 horas $(4 \%)$ depois disso, o aumento observado foi muito baixo (cerca de 1\%). Por outro lado, não se obteve rendimento de glicose para casca de soja e bagaço de cana. 
Figura 2 - Perfil da produção de glicose na sacarificação de resíduos agroindustriais usando extrato bruto enzimático produzido por T. reesei NRRL $3652 \mathrm{em}$ casca de soja.

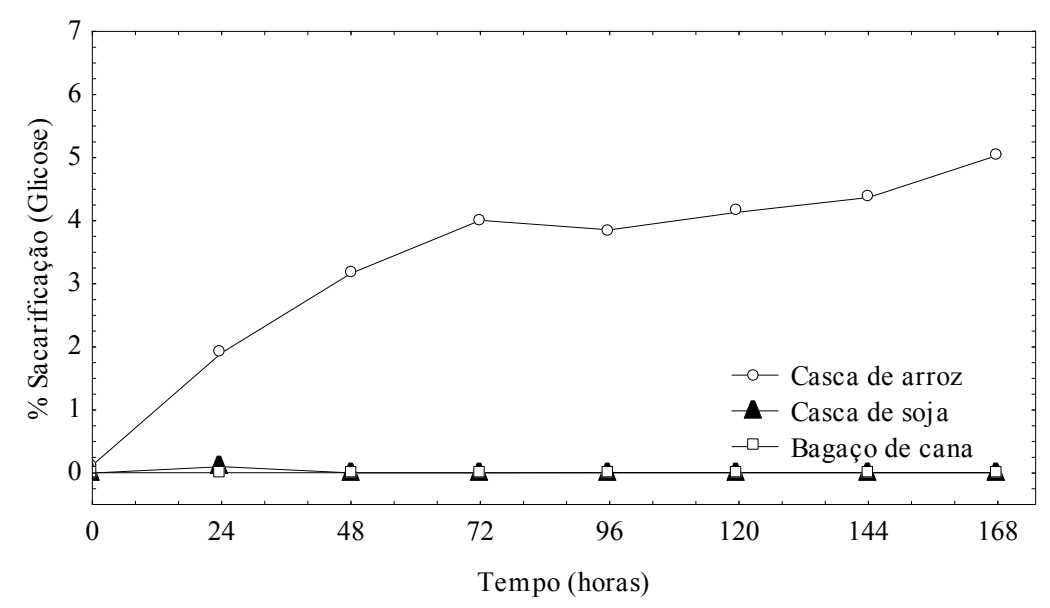

Considerando que a casca de arroz não passou por nenhum tipo de pré-tatamento, esse maior rendimento de glicose pode ser atribuído possivelmente ao pó de arroz que está junto com a casca de arroz e que por sua vez pode conter açúcares que podem ter sido hidrolisados pelas enzimas presentes no extrato enzimático. Segundo Falkoski et al. (2013) a biomassa lignocelulósica não pode ser sacarificada por enzimas com rendimentos elevados sem um processo de pré-tratamento, isso porque, a lignina e a hemicelulose presentes na parede da célula vegetal formam um obstáculo para a ação de enzimas.

A remoção eficiente da lignina é um dos requisitos mais importantes para uma eficaz sacarificação enzimática. A lignina limita a taxa de hidrólise, atuando como um escudo, evitando que as partes hidrolisáveis do substrato sejam hidrolisadas. Além disso, a lignina condensada pode absorver proteínas a partir de soluções aquosas e a remoção de lignina deve melhorar o desempenho da hidrólise pela redução de adsorção não específica das celulases (YANG e WYMAN, 2004).

Yoo et al. (2011) utilizaram a combinação de três celulases comerciais (Celluclast 1,5L, Novozyme 188 e Viscozyme ${ }^{\circledR L}$ ) para a sacarificação enzimática de casca de soja submetida a diferentes pré-tratamentos, e obtiveram um rendimento de glicose de $0,16 \mathrm{~g} \mathrm{~g}^{-1} \mathrm{em}$ casca de soja sem tratamento.

Ortega et al. (2001) estudaram a cinética da sacarificação de celulases de Trichoderma reesei, tal como a concentração de substrato, diversas enzimas, temperatura e $\mathrm{pH}$, e obtendo assim as melhores condições experimentais para degradar celulose padrão (CMC, celulose microgranular e Sigmacell 100).

\section{CONCLUSÃO}

O maior rendimento de açúcares redutores catalisado pelas enzimas presentes no extrato enzimático bruto foi obtido em casca de soja. No entanto, este valor poderá ser melhorado com o estudo de diferentes tipos de pré-tratamentos em diferentes resíduos lignocelulósicos. O processo de produção de etanol de segunda geração a partir de diferentes tipos de resíduos 
agrícolas é uma estratégia que diminuiria os impactos ambientais. Assim, novos avanços para este processo devem ser alcançados para torná-lo mais eficaz e uma realidade sustentável.

\section{AGRADECIMENTOS}

À URI - Campus de Erechim pela infraestrutura e a CAPES pelo apoio financeiro.

\section{REFERÊNCIAS}

DESWAL, D.; KHASA, Y. P.; KUHAD, R. C, Optimization of cellulase production by a brown rot fungus Fomitopsis sp. RCK2010 under solid state fermentation. Bioresour. Technol., v. 102, p. 6065-6072, 2011.

FALKOSKI, D. F.; GUIMARÃES, V. M.; ALMEIDA, M. N.; ALFENAS, A. C.; COLODETTE, J. L.; REZENDE, S. T., Chrysoporthe cubensis: A new source of cellulases and hemicellulases to application in biomass saccharification processes. Bioresour. Technol., v. 130, p. 296-305, 2013.

JUTURU, V.; WU, J. C., Microbial cellulases: Engineering, production and applications. Renew. Sust. Energ. Rev., v. 33, p. 188-203, 2014.

KESTON, A. Paper 31C, $129^{\text {th }}$ Meeting of the American Chemical Society, April, 1956.

LIU, D.; ZHANG, R.; YANG, X.; WU, H.; XU, D.; TANG, Z.; SHEN, Q., Thermostable cellulase production of Aspergillus fumigatus Z5 under solid-state fermentation and its application in degradation of agricultural wastes. Int. Biodeterior. Biodegradation, v. 65, p. 717-725, 2011.

MILLER, G. L., Use of dinitrosalisylic acid reagent for determination of reducin sugar. Anal. Chem., v.31, p. 426 - 428, 1959.

ORTEGA, N.; BUSTO, M. D.; PEREZ-MATEOS, M., Kinetics of cellulose saccharification by Trichoderma reesei cellulases. Int. Biodeter. Biodegr., v. 47, p. 7-14, 2001.

RAGHUWANSHI, S.; DESWAL, D.; KARP, M.; KUHAD, R. C., Bioprocessing of enhanced cellulase production from a mutant of Trichoderma asperellum RCK2011 and its application in hydrolysis of cellulose. Fuel, v. 124, p. 183-189, 2014.

SANCHEZ, O. J.; CARDONA, C. A., Trends in biotechnological production of fuel ethanol from different feedstocks. Bioresour. Technol., v. 99, p. 5270-5295, 2008.

SUKUMARAN, R. K.; SINGHANIA, R. R.; MATHEW, G. M.; PANDEY A., Cellulase production using biomass feed stock and its application in lignocellulose saccharification for bioethanol production. Renew. Energ., v. 34, p.421-428. 2009.

YANG, B.; WYMAN, C. E., Effect of xylan and lignin removal by batch and flowthrough pretreatment on the enzymatic digestibility of corn stover cellulose. Biotechnol. Bioprocess Eng., v. 86, 88-95, 2004.

YOO, J.; SAJID ALAVI, S.; PRAVEEN VADLANI, P.; AMANOR-BOAD, V., Thermomechanical extrusion pretreatment for conversion of soybean hulls to fermentable sugars, Bioresour. Technol., v. 102, p. 7583- 7590, 2011. 